\title{
Raman optical activity using twisted photons
}

\author{
Kayn A. Forbes* \\ School of Chemistry, University of East Anglia, Norwich NR4 7TJ, United Kingdom
}

\begin{abstract}
Raman optical activity underpins a powerful vibrational spectroscopic technique for obtaining detailed structural information about chiral molecular species. The effect centers on the discriminatory interplay between the handedness of material chirality with that of circularly-polarized light. Twisted light possessing an optical orbital angular momentum carries helical phase fronts that screw either clockwise or anticlockwise, and thus possess a handedness that is completely distinct from the polarization. Here a novel form of Raman optical activity that is sensitive to the handedness of the incident twisted photons through a spin-orbit interaction of light is identified, representing a new chiroptical spectroscopic technique.
\end{abstract}

\section{*k.forbes@uea.ac.uk}

Introduction - The Raman effect underpins powerful spectroscopic techniques that have been extensively utilised throughout the sciences [1-4]. Raman scattering is the inelastic scattering of incident light, which after a Stokes event may leave a molecule in an excited vibrational state whilst shifting the scattered photon to a lower frequency. This type of Raman scattering forms the basis behind Raman vibrational spectroscopy. An additional and distinct attribute is that for chiral molecules the Raman effect can measure a small difference in the intensity of scattered right- and leftcircularly polarized light. This contribution to the overall Raman scattering process is known as Raman optical activity (ROA) [5], and due to the fact the vast majority of biomolecules found throughout nature are chiral, this chiroptical sensitivity has been requisite in determining their structure, conformations, and functionalities [6,7]. Importantly, ROA persists in aqueous solutions and therefore is applicable to a wide range of biological macromolecules, namely proteins, carbohydrates, nucleic acids and viruses [8-11].

Natural ROA is what is termed a chiroptical effect: a light-matter interaction between a chiral molecule and light that has a sensitivity to the handedness of both the material and optical components [12]. Generally, chiroptical effects are manifest through the interplay of molecular handedness with right- and left-circularly-polarized light [13]. Well known examples of such phenomena include circular dichroism (CD), circular differential Rayleigh and Raman scattering, optical rotation (OR), and the discriminatory forces which can occur in optical $[14,15]$ and intermolecular forces [16]. Molecular handedness stems from identical chiral molecules which exist in two mirror-image forms known as enantiomers that are nonsuperimposible by translations and rotations - see Figure 1. The handedness of circularly-polarized light originates from the helical structure that the electric (and magnetic) field vector traces out when propagating. These polarizations can be either left- or right-handed depending whether they rotate anticlockwise or clockwise, respectively, and are denoted by the helicity eigenvalues $\sigma(\mathrm{L} / \mathrm{R})= \pm 1$, conveying a spin angular momentum (SAM) of $\pm \hbar$ per photon. In an analogous fashion, the class of light beams that carry an optical orbital angular momentum (OAM) of $\pm \ell \hbar$ per photon (where $\ell$ denotes the integer-valued topological charge or winding number), due to their helical phase front structure, are manifestly chiral. These twisted light beams (or optical vortices) screw to the left for $\ell>0$ and to the right for $\ell<0$. Since their discovery, these structured light beams have predominantly been utilised in optical communications and particle manipulation, as well as information transfer, imaging, and fundamental entanglement studies [17-22].

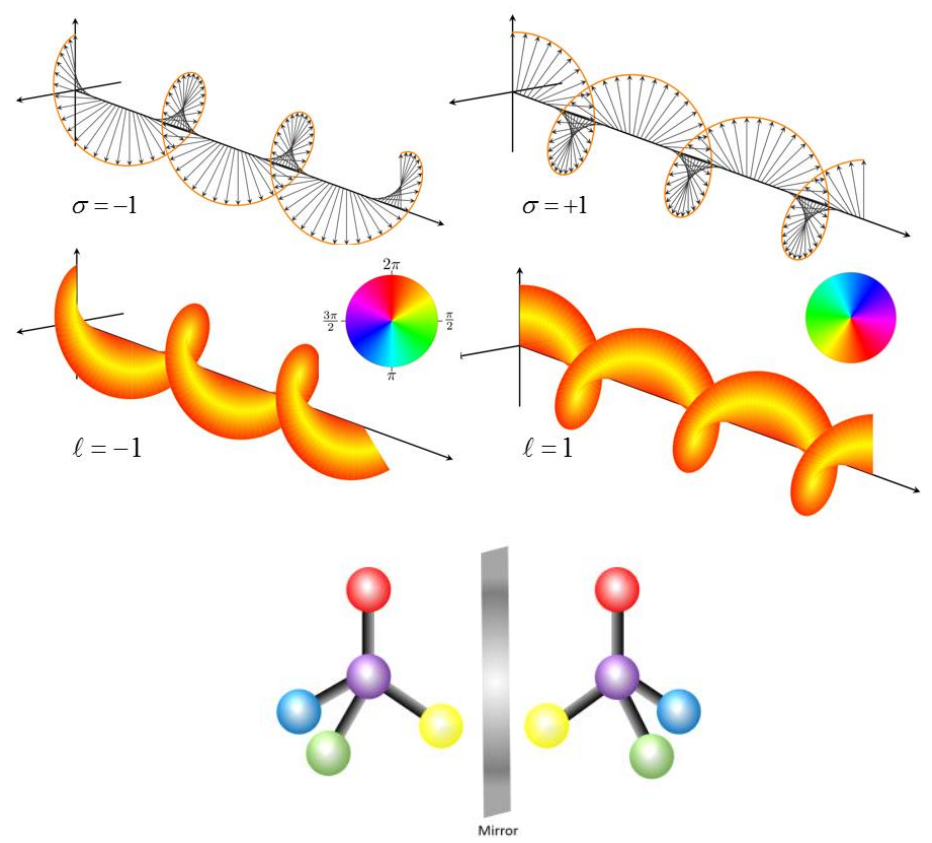

Figure 1 (color online) Different forms of handedness; circularly polarized light, twisted light with azimuthal phase, and a pair of nonsuperimposable molecular enantiomers.

The question naturally arises whether we can expect to see chiroptical effects in chiral matter dependent on the phase front handedness (i.e. the sign of $\ell$ - which is completely distinct form the polarization handedness) of the twisted photons. This issue has spawned a nascent field of research that is growing rapidly, with the majority of studies utilising exotic experimental setups involving plasmonic effects or helicity-dependent intensity distributions of 
non-paraxial light [23-30]. Only recently has a mechanism been elucidated for paraxially propagating light beams interacting with standard dielectric molecular matter [31,32]. In these quantum electrodynamical (QED) studies a form of natural circular dichroism, the differential absorption of left- and right-handed circularly polarized light, was discovered and termed circularvortex dichroism (CVD). Of fundamental importance was the identification of the necessity of engaging electric quadrupole (E2) coupling if one wishes to observe a chiroptical sensitivity to vortex handedness in these interactions. The underlying mechanism being that E2 transition moments interact with the gradient of the electric field, and this introduces longitudinal components to the interaction and thus allow for a spin-orbit interaction (SOI) of light [33]: a dependence on the product of helicity and topological charge $\sigma \ell$. There have been a very limited number of studies on molecular spectroscopic applications of twisted light [34-37]. The experiments do, however, appear to suggest that $\ell$ can play a chiroptical role as theoretically predicted by the QED studies. In this Letter we highlight a novel form of ROA where circularly-polarized Laguerre-Gaussian photons are scattered at different rates by chiral molecules, introducing the potential for a new spectroscopic technique based on twisted light.

Circular-vortex differential scattering (CVDS) - The most important contribution to scattering is a two photon event: the annihilation of an input photon and the creation of an output photon. In a theory based on photons interacting with multipolar moments [37], each photon annihilation or creation event involves a coupling of an electric dipole (E1), electric quadrupole (E2), or magnetic dipole transition moment (M1) with the electromagnetic field. It has already been explicitly highlighted in previous studies how E1 and M1 couplings to chiral molecules cannot produce optical processes that discriminate the sign of $\ell$ [38], and therefore for our purposes we can neglect the M1 term along with the E1M1 interferences in the ensuing derivation, the latter being responsible for the wellknown chiroptical ' $G$ ' tensor.

Our initial system state consists of $n$ Laguerre-Gaussian (LG) photons in the mode $(k, \eta, \ell, p)-k$ is the wave vector, $\eta$ is polarization, $\ell$ winding number, $p$ radial index - and a molecule in the ground state $|n(k, \eta, \ell, p)\rangle\left|E_{0}(\xi)\right\rangle$; the final state of the system is $|n-1(k, \eta, \ell, p)\rangle\left|1\left(\boldsymbol{k}^{\prime}, \eta^{\prime}\right)\right\rangle\left|E_{\mathrm{m}}(\xi)\right\rangle$ where the scattered photon is in some mode $\left(\boldsymbol{k}^{\prime}, \eta^{\prime}\right)$. Using textbook quantum electrodynamical methods [39], including diagrammatic (see Figure 2) second-order time-dependent perturbation theory, and the vacuum electromagnetic mode expansions for LG beams [40], casts the matrix element as:

$$
\begin{aligned}
M_{f i} & =-\left(\frac{\hbar c}{4 \varepsilon_{0} V}\right) \sqrt{k k^{\prime} n} f_{|\ell|, p}(r) \vec{e}_{i}^{\prime} e_{k} \\
& \times\left(\alpha_{i k}^{m 0}+\left(\frac{i \ell \hat{\phi}_{l}}{r}+i k \hat{z}_{l}\right) A_{i k l}^{m 0}-i k_{l}^{\prime} A_{k i l}^{m 0}\right) \mathrm{e}^{-i k^{\prime} \cdot r} \mathrm{e}^{i k z} \mathrm{e}^{i \ell z},
\end{aligned}
$$

where the Einstein rule for summation over repeated indices is implicit; $\boldsymbol{e}$ and $\boldsymbol{e}^{\prime}$ are the polarization vectors of the incident and emitted photons respectively; $V$ is the quantization volume and $f_{|\ell|, p}(r)$ is a radial distribution function, dependent on the absolute value of the topological charge $|\ell|$ and not its sign. The E1 contributions are manifest through $\alpha_{i k}^{m 0}\left(\omega,-\omega^{\prime}\right)$, the well-known frequency-dependent molecular polarizability for electric-dipole interactions

$\alpha_{i k}^{m 0}\left(\omega,-\omega^{\prime}\right)=\sum_{p}\left(\frac{\mu_{i}^{m p} \mu_{k}^{p 0}}{E_{p 0}-\hbar c k}+\frac{\mu_{k}^{m p} \mu_{i}^{p 0}}{E_{p 0}+\hbar c k^{\prime}}\right)$,

and the E1E2 contributions through $A_{i k l}^{m 0}\left(\omega,-\omega^{\prime}\right)$, the corresponding electric-dipole-electric-quadrupole molecular response tensor

$$
A_{i k l}^{m 0}\left(\omega,-\omega^{\prime}\right)=\sum_{p}\left(\frac{\mu_{i}^{m p} Q_{k l}^{p 0}}{E_{p 0}-\hbar c k}+\frac{Q_{k l}^{m p} \mu_{i}^{p 0}}{E_{p 0}+\hbar c k^{\prime}}\right) .
$$

In deriving (1) we have utilised the following result [31]

$$
\begin{aligned}
\mathrm{E} 2 & =-Q_{i j}(\xi) \nabla_{j} e_{i}^{\perp}\left(\boldsymbol{R}_{\xi}\right) \approx-Q_{i j}(\xi) \nabla_{j} f_{|\ell|, p}(r) \mathrm{e}^{(i k z+i \ell \phi)} \\
& \approx-Q_{i j}(\xi) f_{|| \mid, p}(r)\left[\frac{i \ell \hat{\phi}_{j}}{r}+i k \hat{z}_{j}\right] \mathrm{e}^{(i k z+i \ell \phi)}
\end{aligned}
$$

where $e_{i}^{\perp}\left(\boldsymbol{R}_{\xi}\right)$ is the vacuum electric field mode expansion operator $Q_{i j}(\xi)$ is the electric quadrupole transition moment operator, and we have neglected the associated terms dependent on $\hat{\boldsymbol{r}}$ as they do not contribute to the CVDS effect.

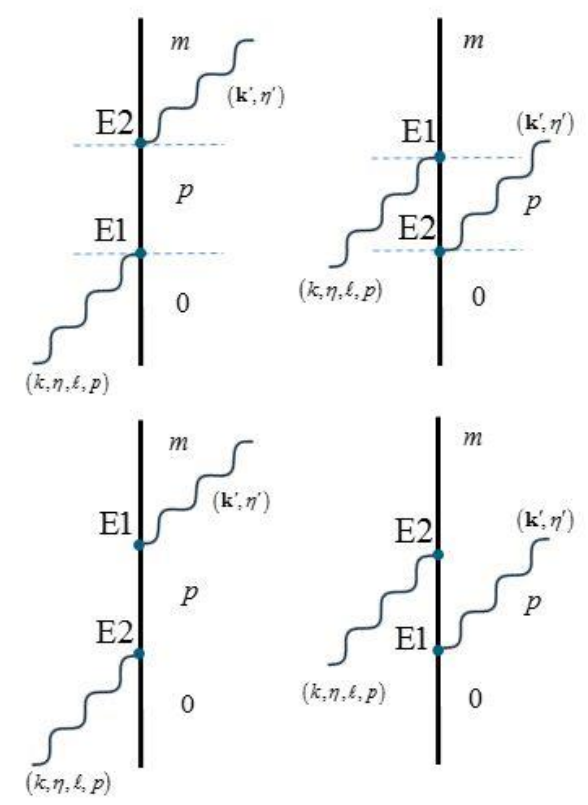


Figure 2 (color online) Time-ordered Feynman graphs for calculating the E1E2 contribution to the quantum amplitude for scattering of a twisted photon of mode $(k, \eta, \ell, p)$. Time progresses vertically and the blue-dashed horizontal lines demarcate the boundaries between initial, intermediate, and final system states. The E1E1 contribution requires only one pair of graphs, with the E2 vertices replaced by E1 interactions.

Raman scattering constitutes an optical process with a different initial and final state for the total light-matter system. Therefore the physical observable occurs as a rate (rather than an energy or force [41]), and can be calculated with the aid of the well-known Fermi rate rule. The Fermi rule - $\Gamma=2 \pi \hbar^{-1} \rho_{f}\left|M_{f i}\right|^{2}$ - requires the modulus square of the matrix element (1) (along with the density of final states $\rho_{f}$ ). This will produce terms involving an $|\alpha|^{2}-, \alpha A$ and $|A|^{2}$-dependence. Chiral molecules can support all of these contributions to scattering, though optical activity only manifests in $\alpha A$ scattering; achiral molecules can only support the nondiscriminatory $|\alpha|^{2}$ and $|A|^{2}$. The term dependent upon the square of the molecular polarizability $|\alpha|^{2}$ is simply the result for standard Raman scattering, which has no dependence upon either the handedness of the matter, polarization, or topological charge. The $|A|^{2}$ terms are neglected as they are significantly smaller than both $|\alpha|^{2}$ and $\alpha A$ contributions, though have been shown to provide chirality-independent information about the shape of a scatterer [42]. This leaves us to concentrate on the $\alpha A$ interference terms, which can only be supported by chiral molecules, changing sign as one chiral molecule is replaced by its enantiomer:

$\Gamma^{(\alpha A)}=-\kappa \operatorname{Re} \vec{e}_{i} e_{j}^{\prime} e_{k} \bar{e}_{m}\left[\left(\frac{i \ell \hat{\phi}_{l}}{r}+i k \hat{z}_{l}\right) \alpha_{i k}^{m 0} A_{j m l}^{m 0}-i k_{l}^{\prime} \alpha_{i k}^{m 0} A_{m j l}^{m 0}\right]$,

where $\kappa=n \pi \hbar k k^{\prime} \rho_{f} c^{2} f_{|\ell|, p}^{2}(r) / 2 \varepsilon_{0}^{2} V^{2}$. Clearly in the first term in square brackets we have produced a term dependent linearly on $\ell$ and hence its sign. Currently the right-hand side of (5) represents an unphysical imaginary or complex-value quantity, and therefore to produce a real observable result we may invoke circularly-polarized photon through the relation [39] $e_{k}^{\mathrm{L} / \mathrm{R}} \bar{e}_{m}^{\mathrm{L} / \mathrm{R}}=\frac{1}{2}\left[\left(\delta_{k m}-\hat{k}_{k} \hat{k}_{m}\right) \mp i \varepsilon_{k m n} \hat{k}_{n}\right]$, which allows us to write the differential rate $\tilde{\Gamma}\left(\mathrm{R} \rightarrow \eta^{\prime}\right)-\tilde{\Gamma}\left(\mathrm{L} \rightarrow \eta^{\prime}\right)$ as

$$
\Delta \tilde{\Gamma}^{(\mathrm{R}(\sigma)-\mathrm{L}(\sigma))}=\kappa \vec{e}_{i}^{\prime} e_{j}^{\prime} \varepsilon_{k m n} \hat{k}_{n}\left[\left(\frac{\ell \hat{\phi}_{l}}{r}+k \hat{z}_{l}\right) \alpha_{i k}^{m 0} A_{j m l}^{m 0}-k^{\prime} \hat{k}_{l} \alpha_{i k}^{m 0} A_{m j l}^{m 0}\right],
$$

where $\varepsilon_{k m n}$ is Levi-Civita tensor which is fully antisymmetric with respect to its indices. We have now derived the rate of circular- vortex differential scattering (CVDS): in (6), the term dependent on $\ell$ is sensitive to both the helicity of light and the sign of $\ell$. At this juncture the spin-orbit interaction of light $[33,31]$ (SOI) becomes obvious: without the introduction of circularly-polarized light (SAM), the term dependent on the topological charge and unique to Laguerre-Gaussian mode light (OAM) would remain imaginary and not be able to engage as an observable effect. It must be made clear however that only this single term is representative of an SOI; the other two terms are the well-known mechanisms behind ROA. We may explicitly highlight the $\sigma \ell$-dependent SOI term:

$\tilde{\tilde{\Gamma}}=\sigma \ell \kappa \overrightarrow{e_{i}} e_{j}^{\prime} \varepsilon_{k n m} \hat{k}_{n} \frac{\hat{\phi}_{l}}{2 r} \alpha_{i k}^{m 0} A_{j m l}^{m 0}$,

The CVDS effect exhibits distinct and novel behaviours: firstly, for a fixed molecular handedness and sign of $\ell$ ( $\ell>0$ for example), the CVDS contribution is different for a different handedness of circular-polarization - we have now explicitly provided the underlying mechanism and explanation for the exploratory experimental results reported in [36]. Important to this behaviour is how the CVDS effect, in comparison to standard ROA, appears to increase in magnitude for an increasing value of $\ell$, representing a unique ability to enhance the magnitude of the overall ROA signal. Secondly and of potentially more interest, is the discriminatory behaviour of the CVDS effect with regards to the sign of $\ell$ : the CVDS contribution differs for $\ell>0$ vs $\ell<0$. Interestingly, for the particular case of $\ell=-1$ it would appear to markedly decrease the total ROA.

Vibrational CVDS - Up until this point, the rate (6) has been derived in a general form applicable to either Rayleigh (elastic) or Raman (inelastic) scattering. Raman scattering predominantly involves molecular vibrational or rotational transitions (or phonons in condense matter). Vibrational ROA molecular spectroscopy offers distinct advantages, such as it does not require chromophores, and can probe all the fundamental $(3 N-6)$ fundamental molecular vibrational transitions (where $N$ is the number of atoms). As such, we now look to develop (6) into a form that pertains to Raman scattering and that is most applicable to real experimental observations. To proceed, we employ the adiabatic BornOppenheimer approximation to describe the molecular states. In vibrational Raman scattering the molecule remains in the ground electronic state for the initial and final state of process, but changes its vibrational state from $v \rightarrow v^{\prime}$ In place of $\left|E_{0}(\xi)\right\rangle$, the initial and final molecular states are now represented by $\left|\psi_{0}\left(q_{\mathrm{e}}, q_{\mathrm{n}}\right)\right\rangle\left|\chi_{0 v}\left(q_{\mathrm{n}}\right)\right\rangle$ and $\left|\psi_{0}\left(q_{\mathrm{e}}, q_{\mathrm{n}}\right)\right\rangle\left|\chi_{0 v^{\prime}}\left(q_{\mathrm{n}}\right)\right\rangle$, respectively. The molecular wavefunction has been factorised into a ground electronic state $\psi_{0}\left(q_{\mathrm{e}}, q_{\mathrm{n}}\right)$ and vibrational state $\chi_{0 v}\left(q_{\mathrm{n}}\right)$. Here $q_{\mathrm{e}}$ and $q_{\mathrm{n}}$ denote the electronic and nuclear coordinates, respectively. The electronic state has a parametric dependence on nuclear coordinates, and the vibrational state refers to the $v$ th level of an oscillator for the ground 
electronic state in one of the coordinates $q_{\mathrm{n}}$. With the above taken into consideration, it is well highlighted how the molecular response tensors $\alpha_{i k}^{m 0}$ (Eq. (2)) and $A_{i k l}^{m 0}$ (Eq. (3)) can be written as [12,39]

$\left\langle\chi_{0 v^{\prime}}\left|\alpha_{i k}^{00}\right| \chi_{0 v}\right\rangle=\alpha_{i k}^{v^{\prime} v} ; \quad\left\langle\chi_{0 v^{\prime}}\left|A_{i k l}^{00}\right| \chi_{0 v}\right\rangle=A_{i k l}^{v^{\prime} v}$,

where $\alpha_{i k}^{00}$ and $A_{i k l}^{00}$ are the standard frequency-dependent molecular response tensors (2) and (3) (for $m=0$ ), respectively, that possess a dependence on $q_{\mathrm{n}}$ through $\mu_{i}^{0 p}$ and $Q_{i j}^{0 p}$. The optically active Raman intensities may be calculated in the usual way through the product of Placzek's expansions for the molecular response tensors (8) in the normal vibrational coordinates [12]. The approximations used to derive (8) involve the choice that the incident light is nonresonant, and therefore vibrational energy differences are neglected and $k=k^{\prime}$. In this approximation $\alpha_{i k}^{v^{\prime} v}$ is index-symmetric, and $A_{i k l}^{v^{\prime} v}$ is $k l$-symmetric. The results can easily be extended to near-resonant conditions by reversing these approximations, and the molecular response tensors contribute an antisymmetric as well as symmetric part.

Taking all of the above into consideration and accounting for the density of final states [39] as $\rho_{f}=k^{\prime 2} \mathrm{~d} \Omega^{\prime} V /(2 \pi)^{3} \hbar c$ allows the result to be expressed in terms of an observable: the difference in scattered radiant intensity $I\left(\mathrm{R} \rightarrow \eta^{\prime}\right)-I\left(\mathrm{~L} \rightarrow \eta^{\prime}\right)$ (i.e. the difference in energy radiated in the direction $\hat{\boldsymbol{k}}^{\prime}$ per unit solid angle per unit time) for $N$ molecules in the initial vibrational state $v$ :

$$
\begin{aligned}
\Delta I\left(\boldsymbol{k}^{\prime}\right) & =\frac{N_{v} I_{0} k^{4}}{16 \pi^{2} \varepsilon_{0}^{2}} f_{\mid \ell, p}^{2}(r) \bar{e}_{i}^{\prime} e_{j}^{\prime} \varepsilon_{k m n} \hat{k}_{n} \\
& \times\left[\left(\frac{\ell \hat{\phi}_{l}}{r}+k \hat{z}_{l}\right) \alpha_{i k}^{m 0} A_{j m l}^{m 0}-k \hat{k}_{l} \alpha_{i k}^{m 0} A_{m j l}^{m 0}\right],
\end{aligned}
$$

where $I_{0}$ is the input irradiance, dependent on the radial distribution function. The difference in scattered radiant intensity (9) as it stands currently is applicable to systems with molecular orientational order; a key facet to ROA, and its major advantage over x-ray crystallography methods, is its ability to study molecules in aqueous solution. For the theory to account for molecular liquids (and gases) we require the total orientational average of (9). The necessary scheme involves the $5^{\text {th }}$-rank average $\left\langle\alpha_{i k}^{v^{\prime} v}(\omega) A_{j m l}^{v^{\prime} v}(\omega)\right\rangle$, and using standard techniques [43] we obtain

$$
\begin{aligned}
\left\langle\Delta I\left(\boldsymbol{k}^{\prime}\right)\right\rangle= & \frac{N_{v} I_{0} k^{5}}{480 \pi^{2} \varepsilon_{0}^{2}} f_{|\ell|, p}^{2}(r) \varepsilon_{\mu \pi \sigma} \alpha_{\lambda \mu}^{v^{\prime} v} A_{\pi \lambda \sigma}^{v^{\prime} v}\left[1-3\left(\boldsymbol{e}^{\prime} \cdot \hat{\boldsymbol{k}}\right)\left(\overline{\boldsymbol{e}}^{\prime} \cdot \hat{z}\right)\right. \\
& \left.-3\left(\hat{\boldsymbol{k}}^{\prime} \cdot \hat{\boldsymbol{k}}\right)-\frac{3 \ell}{k r}\left(\boldsymbol{e}^{\prime} \cdot \hat{\boldsymbol{k}}\right)\left(\overline{\boldsymbol{e}}^{\prime} \cdot \hat{\boldsymbol{\phi}}\right)\right]
\end{aligned}
$$

Another important observable quantity, originally introduced by Barron and Buckingham [44], is the dimensionless circular intensity difference (CID) (or incident circular polarization (ICP) ROA) $\Delta=\left(I^{\mathrm{R}}-I^{\mathrm{L}}\right) /\left(I^{\mathrm{R}}+I^{\mathrm{L}}\right)$; the quantity in the numerator represents the ROA effect because the scattering rate of circularlypolarized photons for standard $|\alpha|^{2}$ Raman scattering is the same (and thus subtraction from one another yields zero); the quantity in the denominator is, due to the same logic, dominated by the standard Raman effect for non-resonant light:

$$
\begin{aligned}
\left\langle I^{\mathrm{R}}+I^{\mathrm{L}}\right\rangle & =\frac{N_{v} I_{0} k^{4}}{960 \pi^{2} \varepsilon_{0}^{2}} f_{|\ell|, p}^{2}(r) \\
& \times\left[\left(1-3\left|\hat{\boldsymbol{k}} \cdot \boldsymbol{e}^{\prime}\right|^{2}\right) \alpha_{\lambda \lambda}^{(0) v^{\prime} v} \bar{\alpha}_{\mu \mu}^{(0) v^{\prime} v}+\left(7-\left|\hat{\boldsymbol{k}} \cdot \boldsymbol{e}^{\prime}\right|^{2}\right) \alpha_{\lambda \mu}^{(2) v^{\prime} v} \bar{\alpha}_{\lambda \mu}^{(2) v^{\prime} v}\right],
\end{aligned}
$$

where as is standard, the $\alpha_{i k}^{v^{\prime} v}$ contributions have been split into scalar $\alpha_{\lambda \lambda}^{(0) v^{\prime} v}$ and symmetric $\alpha_{\lambda \mu}^{(2) v^{\prime} v}$ parts. $\langle\Delta\rangle$ for $\alpha A$ contributions is therefore given by the ratio of (10) and (11). The standard results for the E1M1 (or more correctly ' $\alpha G^{\prime}$ ) contribution to $\left\langle\Delta I\left(\boldsymbol{k}^{\prime}\right)\right\rangle$ are well-known $[39,12]$, and can be easily altered to account for LG modes and inserted into (10) to give the total $\langle\Delta\rangle$ - remembering however that they do not depend on the sign of $\ell$ and therefore do not contribute to the CVDS effect.

Scattering Geometry - we now propose two experimental scattering setups for observing the CDVS effect: the first involves studying the scattering at right-angles $\theta=90^{\circ}$ (see Figure 3), and resolving the polarization at $45^{\circ}$; or alternatively studying the scattering at an arbitrary angle $\theta$ without resolving the polarization.

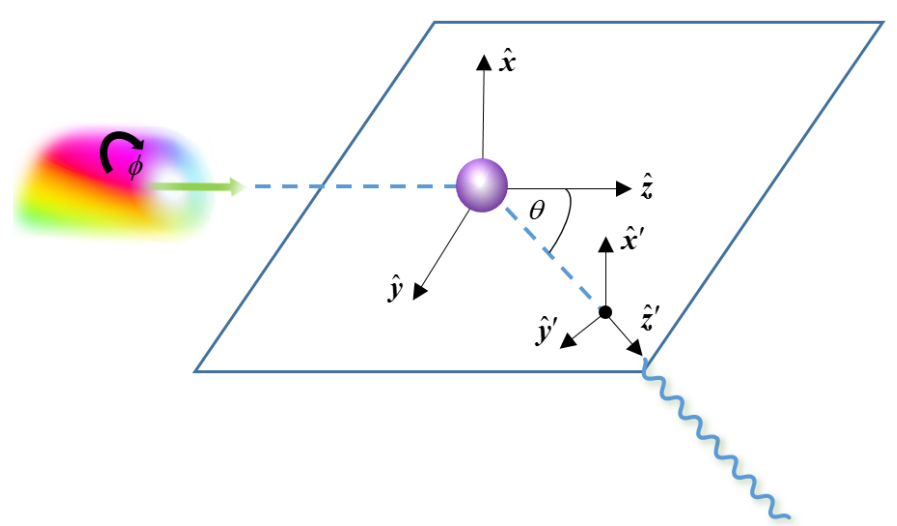

Figure 3 (color online) Scattering geometry for an incident LG vortex photon described by the unit vectors $(\hat{\boldsymbol{x}}, \hat{\boldsymbol{y}}, \hat{\boldsymbol{z}})$ scattered by a molecule (purple sphere) at a scattering angle $\theta$. The unit vectors $\left(\hat{\boldsymbol{x}}^{\prime}, \hat{\boldsymbol{y}}^{\prime}, \hat{\boldsymbol{z}}^{\prime}\right)$ describe the scattered photon (wavy line).

We need only make use of the following coordinate transformation $\hat{\boldsymbol{\phi}}=-\hat{\boldsymbol{x}} \sin \phi+\hat{\boldsymbol{y}} \cos \phi$ as $\hat{\boldsymbol{z}}$ is identical in either Cartesian or cylindrical coordinates and we have no terms dependent on $\hat{\boldsymbol{r}}$. The 
first example requires $\boldsymbol{e}^{\prime}=\left(\hat{\boldsymbol{x}}^{\prime}+\hat{\boldsymbol{y}}^{\prime}\right) / \sqrt{2}, \quad \hat{\boldsymbol{k}}\left(\hat{\boldsymbol{k}}^{\prime}\right)=\hat{z}\left(\hat{z}^{\prime}\right)$ and $\theta=90^{\circ}$, using the relations $\hat{\boldsymbol{x}}^{\prime}=\hat{\boldsymbol{x}} ; \hat{\boldsymbol{y}}^{\prime}\left(\hat{z}^{\prime}\right)=\hat{\boldsymbol{y}}(\hat{z}) \cos \theta \mp \hat{z}(\hat{\boldsymbol{y}}) \sin \theta$ , we see that the differential circular scattered intensity for polarization resolved at $45^{\circ}$ :

$$
\left\langle\Delta I_{45^{\circ}\left(e^{\prime}\right)}^{90^{\circ}\left(k^{\prime}\right)}\right\rangle=-\frac{N_{v} I_{0} k^{5}}{960 \pi^{2} \varepsilon_{0}^{2}} f_{|\ell|, p}^{2}(r) \varepsilon_{\mu \pi \sigma} \alpha_{\lambda \mu}^{v^{\prime} v^{\prime}} A_{\pi \lambda \sigma}^{v^{\prime \prime} v}\left[1+\frac{3 \ell}{k r} \sin \phi\right] .
$$

Under the same assumptions, the radiant intensity difference for an arbitrary scattering angle is

$$
\begin{aligned}
\left\langle\Delta I\left(\boldsymbol{k}^{\prime}\right)\right\rangle & =\frac{N_{v} I_{0} k^{5}}{480 \pi^{2} \varepsilon_{0}^{2}} f_{|\ell|, p}^{2}(r) \varepsilon_{\mu \pi \sigma} \alpha_{\lambda \mu}^{v^{\prime} v} A_{\pi \lambda \sigma}^{v^{\prime} v} \\
& \times\left[1-3 \sin ^{2} \theta-3 \cos \theta+\frac{3 \ell}{2 k r} \sin 2 \theta \cos \phi\right] .
\end{aligned}
$$

For $\theta=0^{\circ}, 90^{\circ}, 180^{\circ}, \ldots$ the CVDS term in (13) is zero, and we recover the well-known results for light beams with $\ell=0$; however, of more interest is the fact that the function $\sin 2 \theta$ over the range 0 $2 \pi$ produces a scattering spatial distribution that exhibits the intrinsic symmetry of a quadrupole, and we can conclude that the CVDS effect in this scattering geometry is at a maximum when $\theta=45^{\circ}, 135^{\circ}, \ldots$, and clearly differs from the intensity distribution associated with conventional ROA - which comes from the first three terms in square brackets in (13).

An important aspect of observing CVDS ROA will be placing the incident beam off-axis, because in both expressions (12) and (13), the full beam profile integration involving the limit $0-2 \pi$ over the azimuthal angle delivers a null result. In fact, the maximum effect will be observed for in both cases when half of the beam is incident on the sample. The results (12) and (13) can be easily used to calculate their corresponding $\operatorname{CID}\langle\Delta\rangle$.

Common to both CVDS terms in (12) and (13) is the unique dependence on $\ell$ responsible for the effect, but also an exclusive dependence on the factor $(k r)^{-1}$ compared to both standard CDS ROA and Raman scattering (11). Assuming the molecules are within a non-zero beam intensity region, when $k r \approx 1$ and $|\ell|=1$, the CVDS ROA effect is of a similar magnitude to the standard CDS ROA effect, thus increasing the overall magnitude of ROA. As stated earlier, the unique dependence on $\ell$ means CVDS becomes larger in magnitude for higher winding numbers, which theoretically can assume an integer value up to $\pm \infty$. When $k r \ll 1$ (which for a fixed wavelength means the molecules are closer to the intensity singularity of the beam), the CVDS will dominate the other ROA effects, and equally molecules centered further from the center of the beam will contribute less to the effect. However, it must be remembered that the intensity of the incident beam is highlydependent on different parameters, most importantly $r$, through the radial distribution function, and specifically is zero at the center.
Besides generating the CVDS ROA effect itself, the numerous intricate and experimentally controllable properties of structured light carrying optical OAM proffer much scope for enhancing the information that may be gained from ROA. The potential of enhanced ROA through the CVDS mechanism represents an avenue for time-resolved ROA spectroscopy [45] or an alternative to surface-enhanced ROA [46,47] for increasing the typically small ROA effect. Of perhaps even further interest would be the scope for incorporating surface plasmon enhancements with the CVDS ROA. It is also worth bearing in mind that relatively enhanced interactions can take place between E2 moments and twisted light beams [4850].

Conclusion - a new form of ROA which is sensitive to the sign of the topological charge $\ell$ of an incident optical-vortex beam, as well as the polarization and molecular handedness, has been explicitly derived. A manifestation of an SOI in paraxial light with optical SAM and OAM, it has been highlighted how these structured beams deliver a unique ROA mechanism dependent on the product $\sigma \ell$ when compared to standard laser beams in ROA spectroscopy. Alongside offering a multitude of new physics, the CVDS contribution to ROA is seen to be able to dominate the standard ROA effect in certain circumstances, enabling an increased relative magnitude to the background standard Raman scattering. The novel CVDS mechanism highlighted in this work represents the potential for the development of a new spectroscopic technique that synergises the two completely distinct fields of structured light/optical OAM and ROA spectroscopy.

The author would like to thank Dale Green for help producing the figures and David L. Andrews for encouragement and many stimulating discussions.

[1] R. L. McCreery, Raman Spectroscopy for Chemical Analysis (John Wiley \& Sons, 2005).

[2] A. C. Ferrari and D. M. Basko, Nat. Nanotechnol. 8, 235 (2013).

[3] A. C. S. Talari, Z. Movasaghi, S. Rehman, and I. U. Rehman, Appl. Spectrosc. Rev. 50, 46 (2015).

[4] K. Buckley and A. G. Ryder, Appl. Spectrosc. 71, 1085 (2017).

[5] L. D. Barron, S. T. Mutter, and E. W. Blanch, in Chiral Anal. Second Ed. (Elsevier, 2018), pp. 249-291.

[6] V. Parchaňskỳ, J. Kapitan, and P. Bouř, RSC Adv. 4, 57125 (2014).

[7] L. D. Barron, Biomed. Spectrosc. Imaging 4, 223 (2015).

[8] L. D. Barron, L. Hecht, E. W. Blanch, and A. F. Bell, Prog. Biophys. Mol. Biol. 73, 1 (2000).

[9] L. D. Barron, E. W. Blanch, and L. Hecht, Adv. Protein Chem. 62, 51 (2002).

[10] L. D. Barron, E. W. Blanch, I. H. McColl, C. D. Syme, L. Hecht, and K. Nielsen, J. Spectrosc. 17, 101 (2003).

[11] L. A. Nafie, Vibrational Optical Activity: Principles and Applications (John Wiley \& Sons, 2011).

[12] L. D. Barron, Molecular Light Scattering and Optical Activity (Cambridge University Press, 2009).

[13] D. L. Andrews, J. Opt. 20, 033003 (2018). 
[14] D. S. Bradshaw and D. L. Andrews, New J. Phys. 16, 103021 (2014).

[15] K. A. Forbes and D. L. Andrews, Phys. Rev. A 91, 053824 (2015).

[16] A. Salam, Molecular Quantum Electrodynamics: LongRange Intermolecular Interactions (John Wiley \& Sons, New Jersey, 2010).

[17] M. Vasnetsov and K. Staliunas, Optical Vortices (Nova Science Pub Incorporated, 1999).

[18] D. L. Andrews, Structured Light and Its Applications: An Introduction to Phase-Structured Beams and Nanoscale Optical Forces (Academic press, 2011).

[19] A. M. Yao and M. J. Padgett, Adv. Opt. Photonics 3, 161 (2011).

[20] H. Rubinsztein-Dunlop, A. Forbes, M. V. Berry, M. R. Dennis, D. L. Andrews, M. Mansuripur, C. Denz, C. Alpmann, P. Banzer, and T. Bauer, J. Opt. 19, 013001 (2016).

[21] S. M. Barnett, M. Babiker, and M. J. Padgett, Optical Orbital Angular Momentum (The Royal Society, 2017).

[22] M. Babiker, D. L. Andrews, and V. E. Lembessis, J. Opt. 21, 013001 (2019).

[23] X. Zambrana-Puyalto, X. Vidal, and G. Molina-Terriza, Nat. Commun. 5, 4922 (2014).

[24] T. Wu, R. Wang, and X. Zhang, Sci. Rep. 5, 18003 (2015).

[25] W. Brullot, M. K. Vanbel, T. Swusten, and T. Verbiest, Sci. Adv. 2, e1501349 (2016).

[26] A. Afanasev, C. E. Carlson, and M. Solyanik, J. Opt. 19, 105401 (2017).

[27] C. T. Samlan, R. R. Suna, D. N. Naik, and N. K. Viswanathan, Appl. Phys. Lett. 112, 031101 (2018).

[28] S. Wang, Z.-L. Deng, Y. Cao, D. Hu, Y. Xu, B. Cai, L. Jin, Y. Bao, X. Wang, and X. Li, IEEE Photonics J. 10, 1 (2018).

[29] H. Ren and M. Gu, Laser Photonics Rev. 12, 1700255 (2018).

[30] I. V. Reddy, A. Baev, E. P. Furlani, P. N. Prasad, and J. W. Haus, ACS Photonics 5, 734 (2018).

[31] K. A. Forbes and D. L. Andrews, Phys. Rev. A 00, 003800 (accepted) (2019)

[32] K. A. Forbes and D. L. Andrews, Opt. Lett. 43, 435 (2018).

[33] K. Y. Bliokh, F. J. Rodríguez-Fortuño, F. Nori, and A. V. Zayats, Nat. Photonics 9, 796 (2015).

[34] G. Milione, J. Secor, G. Michel, S. Evans, and R. R. Alfano, in Complex Light Opt. Forces V (International Society for Optics and Photonics, 2011), p. 79500H.

[35] J. Li, J. J. Tu, and J. L. Birman, J. Phys. Chem. Solids 77, 117 (2015).

[36] E. Bendau, L. Zhang, R. Gozali, S. Ashrafi, and R. R. Alfano, in Complex Light Opt. Forces XI (Proceedings of SPIE 10120, 2017), p. 1012004.

[37] S. Mamani, L. Shi, T. Ahmed, R. Karnik, A. RodríguezContreras, D. Nolan, and R. Alfano, J. Biophotonics e201800096 (2018).

[38] D. L. Andrews, L. D. Romero, and M. Babiker, Opt. Commun. 237, 133 (2004).

[39] D. P. Craig and T. Thirunamachandran, Molecular Quantum Electrodynamics: An Introduction to RadiationMolecule Interactions (Courier Corporation, 1998).
[40] L. D. Romero, D. L. Andrews, and M. Babiker, J. Opt. B Quantum Semiclassical Opt. 4, S66 (2002).

[41] D. S. Bradshaw and D. L. Andrews, Eur. J. Phys. 38, 034008 (2017).

[42] R. P. Cameron and N. Mackinnon, Phys. Rev. A 98, 013814 (2018).

[43] D. L. Andrews and T. Thirunamachandran, J. Chem. Phys. 67, 5026 (1977).

[44] L. D. Barron and A. D. Buckingham, Mol. Phys. 20, 111 (1971).

[45] K. Hiramatsu, M. Okuno, H. Kano, P. Leproux, V. Couderc, and H. Hamaguchi, Phys. Rev. Lett. 109, 083901 (2012).

[46] T. Wu, X. Zhang, R. Wang, and X. Zhang, J. Phys. Chem. C 120, 14795 (2016).

[47] L. Hu, F. Xi, L. Qv, and Y. Fang, ACS Omega 3, 1170 (2018).

[48] C. T. Schmiegelow and F. Schmidt-Kaler, Eur. Phys. J. D 66, 157 (2012).

[49] M. G. Mandujano and J. A. Maytorena, Phys. Rev. A 88, 023811 (2013).

[50] V. E. Lembessis and M. Babiker, Phys. Rev. Lett. 110, 083002 (2013). 